\title{
Mixing Time and Temporal Starvation of General CSMA Networks with Multiple Frequency Agility
}

\author{
Ka-Kit Lam*†, Chi-Kin Chau ${ }^{\ddagger}$, Minghua Chen*, and Soung-Chang Liew* \\ * Department of Information Engineering, The Chinese University of Hong Kong. \\ $\dagger$ UC Berkeley. $\quad \ddagger$ Massachusetts Institute of Technology. $\S$ Masdar Institute.
}

\begin{abstract}
Mixing time is a fundamental property for a number of transient behaviors of stochastic processes, particularly, random access in CSMA networks. We use mixing time to characterize temporal starvation, which is a transient phenomenon where links can starve for prolonged periods indefinitely often despite having good stationary throughput. Considering a general CSMA network, we study a fundamental setting with multiple frequency agility, such that more than one frequency channel is available, and a link can transmit on at most one of the frequency channels not occupied by its neighbors. The characterization of throughput in such a setting is challenging, involving a hidden Markov chain of the associated stochastic process. This paper develops new results based on the mixing time of hidden Markov chains to shed light on the temporal starvation. Our analytical results quantify the effect of the number of frequency channels on temporal starvation. We provide sufficient and necessary conditions for fast mixing time of the corresponding hidden Markov chain.

Index Terms-CSMA Networks, Distributed Resource Allocation, Transient Behavior, Mixing Time, Temporal Starvation
\end{abstract}

\section{INTRODUCTION}

The study of efficient and fair resource allocation mechanisms in communication networks has been a rich and challenging subject. While the stationary and long-term behavior has been extensively investigated, recent studies have only begun to tackle the more practically relevant but also more difficult problem of temporal and transient behavior in communication networks [3], [1], [5].

In this paper, we study a commonly observed performance issue in CSMA networks, namely temporal starvation. Temporal starvation is intrinsic in CSMA networks, which refers to the phenomenon that links can starve for prolonged periods indefinitely often despite having good stationary throughput. We characterize temporal starvation by the mixing time of the associated throughput process of CSMA networks. We are interested in how the availability of multiple frequency channels will help to alleviate temporal starvation.

We consider the general setting of CSMA networks with multiple frequency agility [8], in which more than one frequency channel is available, and a link can transmit on at most one of the frequency channels not occupied by its neighbors. Although the focus of this paper is on CSMA networks, multiple frequency agility is a basic framework for general distributed resource allocation mechanisms. For instance, a node can access a resource (e.g., a frequency channel) when it is locally detected to be idle and the operation is permitted by policy (e.g., not simultaneously accessing more than one channel). Our study provides a foundation to address the transient behavior of general distributed resource allocation problems ${ }^{1}$.

The main goal of this paper is to understand and quantify the effect of the number of frequency channels on temporal starvation in general CSMA networks. We have several new contributions toward the analytical understanding of multiple frequency agility.

First, we find that the study of temporal starvation in multifrequency CSMA must be done through a hidden Markov chain. This is in contrast to the single frequency scenario, where the temporal starvation is merely characterized by a straightforward Markov chain [3]. In particular, we study the mixing time of a hidden Markov chain considering the activeness of each link rather than the full channel state that not only includes the activeness of the link, but also which frequency it is using when it is active. We remark that the mixing time of hidden Markov chains has not been wellstudied before. Thus, our study not only yields new insights on CSMA networks, but also new techniques for analyzing hidden Markov chains in general.

Second, we quantify the sufficient conditions under which temporal starvation can be significantly alleviated. While it is intuitive that having more channels can alleviate temporal starvation, this is true only when the number of channels is large enough. Our simulation suggests that the number of channels being greater than twice of the maximum degree of the conflict graph (i.e. $q>2 \Delta$ ) is a critical condition for temporal starvation to be substantially alleviated. We confirm this observation by upper bounding mixing time for the corresponding hidden Markov chain analytically. We remark that it is non-trivial to obtain the sufficient conditions of $q>2 \Delta$ 2 .

Third, we show that temporal starvation can be severe if there is insufficient number of channels. We generalize Cheeger's inequality in the context of hidden Markov chains to

\footnotetext{
${ }^{1}$ An immediate application is cognitive radio - nodes dynamically share multiple frequency bandwidth spectrum, subject to complex policy constraints.

${ }^{2}$ In the literature of mixing time[2], characterization of conditions to have fast mixing time is normally challenging. A related problem is random coloring[2](sampling the uniform distribution of graph coloring by Markov chain monte carlo). However, not only is the complete characterization of mixing time for random coloring a wide open question, it is also highly nontrivial even to prove fast mixing for specific graphs with slightly fewer number of colors than twice the maximum degree of the graphs. When compared to mixing time analysis of random coloring, our problem not only has less symmetry but is also related to a less well structured stochastic process (hidden Markov chain).
} 
establish a necessary condition for fast mixing. This necessary condition is on the number of channels. Violating the condition will lead to serious temporal starvation. In particular, we show that if $q<<\Delta$, mixing time for the hidden Markov chain scales exponentially with the number of links under a complete bipartite graph.

In summary, our analytical results includes:

- New tools to upper- and lower- bound the mixing time of hidden Markov chains.

- Sufficient conditions for fast mixing when the number of channel is sufficient.

- An example of slow mixing time when the number of channels is insufficient.

We believe our results add to fundamental understanding of temporal starvation in CSMA networks and the extent to which multi-channel agility may help alleviate it.

\section{A. Motivating Observations}

Before presenting our analytical results, we briefly provide some motivating observations from simulation ${ }^{3}$ (see Fig. 1).

We simulate CSMA with multiple frequency agility on a simple $4 \times 4$ torus network (i.e., a boundaryless $2 \mathrm{D}$ grid). Note that the detailed CSMA model will be introduced next. We test under different numbers of channels and present the data of temporal starvation at 4 different time points (i.e. $t=100,500$, 1000,2000 ). We observe that temporal starvation (measured by the throughput loss as compared to stationary throughput) is significantly reduced, when the number of channels is larger than twice the maximum degree (i.e., 8). Moreover, we observe temporal starvation is severe when there is insufficient number of channels.

Motivated by these observations, we seek to provide a comprehensive understanding of these observations in the following sections.

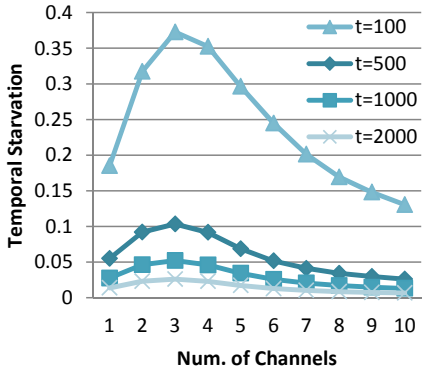

Fig. 1. Temporal starvation vs number of channels, for $4 \times 4$ torus (i.e., a boundaryless 2D grid).

\section{Model ANd Problem Formulation}

\section{A. Basic CSMA Operations}

In a CSMA network with multiple frequency agility, there are $n$ physical links and $q$ frequency channels. Each link has a set of neighboring links. A link can access a channel if and only if the channel is not occupied by its neighboring links, and the link is not using any other channels at the moment. We

\footnotetext{
${ }^{3} \mathrm{~A}$ detailed discussion of simulation can be found in [6]
}

model such a network by a conflict graph $G \triangleq(V, E)$ where $V$ is a set of physical links (each link is a pair of transmitter and receiver in CSMA network), and $E$ is a set of relations representing whether two physical links are neighbors to each other. We let $n \triangleq|V|$. For any $v \in V$, we denote by $\mathcal{N}(v)$ the set of its neighbors. Let $\Delta$ be the maximum degree of the graph, $\Delta \triangleq \max _{v \in V}|\mathcal{N}(v)|$. We assume that all the links have unit capacities. But our results can be generalized to the case of heterogeneous link capacities.

Similar to standard CSMA protocol, we assume that each link maintains a backoff counter for each channel. The backoff value is generated according to an exponential distribution with a certain mean $\frac{1}{\mu_{\mathrm{cd}}}$. The link counts down all the $q$ counters simultaneously at unit rate. When the value of a counter reaches zero, the link transmits on the corresponding channel for a duration that follows another exponential distribution with mean $\frac{1}{\mu_{\mathrm{tx}}}$. During the countdown process, if a link senses a channel being occupied by its neighboring links, it will freeze the corresponding counter, which will be resumed after the channel is released.

\section{B. Throughput Process}

At any given time, a link can be in one of the states, $C \triangleq$ $\{0,1, \ldots q\}$, where state 0 means the link is idle (i.e., not occupying any channels), and state $i>0$ means that the link is actively transmitting on channel $i$. A feasible state of the CSMA network is a vector containing feasible channel states of the links. Denote the set of all feasible states by

$\mathcal{S} \triangleq\left\{x \in C^{n} \mid x(v) \neq x(u)\right.$ or $\left.x(v)=x(u)=0,(u, v) \in E\right\}$ where $x(v) \in C$ represents the channel state of $v$ in CSMA network state $x$.

We can model the evolution of the states of CSMA network with multiple frequency agility by a continuous-time Markov chain $\left(X_{t}\right)_{t>0}$ with $\mathcal{S}$ as the sample space, which we call CSMA-MF. $\left(X_{t}\right)_{t \geq 0}$ is a time-reversible Markov chain with stationary distribution as

$$
\pi(x)=\frac{\prod_{v \in V: x(v) \neq 0} \lambda}{\sum_{x^{\prime} \in \mathcal{S}} \prod_{x^{\prime}(v) \neq 0} \lambda}, \quad x \in \mathcal{S}
$$

where $\lambda \triangleq \frac{\mu_{\mathrm{cd}}}{\mu_{\mathrm{tx}}}$, is referred as link aggressiveness [4].

Note that $\left(X_{t}\right)_{t \geq 0}$ not only captures if a link is transmitting, but also which channel it uses. For link throughput, however, we are only interested in whether the link is transmitting or not. Thus, the link throughput is characterized by a hidden Markov chain of the "link-activeness" on $\left(X_{t}\right)_{t \geq 0}$.

For a state $x \in \mathcal{S}$, we define a mapping $g[x]: \mathcal{S} \mapsto\{0,1\}^{n}$ that maps a state of channels to a state of link-activeness:

$$
g[x](v)= \begin{cases}0, & \text { if } x(v)=0 \\ 1, & \text { if } x(v) \neq 0\end{cases}
$$

Thus, $\left(g\left[X_{t}\right]\right)_{t \geq 0}$ defines a hidden Markov chain for the throughput process of CSMA-MF.

Let $\mathcal{B} \triangleq g[\mathcal{S}]$ be the range of $g$. If $\left(X_{t}\right)_{t \geq 0}$ converges, the hidden Markov chain $\left(g\left[X_{t}\right]\right)_{t \geq 0}$ also converges to its 
corresponding stationary state distribution:

\section{Mixing Time}

$$
\pi\left(g^{-1}[b]\right)=\sum_{x \in \mathcal{S}: g[x]=b} \pi(x), \quad b \in \mathcal{B}
$$

To characterize the transient behavior of CSMA-MF, we rely on the notion of mixing time. We review the background of mixing time in a general setting here.

Let $\mu$ and $\nu$ be probability density functions defined on a sample space $\Omega$. The total variation distance between $\mu$ and $\nu$ is defined by

$$
\|\mu-\nu\|_{T V} \triangleq \max _{A \subset \Omega}|\mu(A)-\nu(A)|
$$

We denote the worst case distance from stationarity for a continuous time Markov chain $\left(X_{t}\right)_{t \geq 0}$ with stationary distribution $\pi$ by

$$
\mathrm{d}(t) \triangleq \max _{x_{0} \in \Omega}\left\|\mathbb{P}\left\{X_{t}=\cdot \mid X_{0}=x_{0}\right\}-\pi(\cdot)\right\|_{T V}
$$

Define a mapping $\tilde{g}: \Omega \rightarrow \tilde{\Omega}$ as a surjective mapping to a smaller sample space $\tilde{\Omega}$. Similarly, we define the worst case distance that captures how far a hidden Markov chain $\left(\tilde{g}\left[X_{t}\right]\right)_{t \geq 0}$ evolves from its stationary state by

$$
\mathrm{d}_{\mathrm{HM}}(t) \triangleq \max _{x_{0} \in \Omega}\left\|\mathbb{P}\left\{\tilde{g}\left[X_{t}\right]=\cdot \mid X_{0}=x_{0}\right\}-\pi\left(\tilde{g}^{-1}[\cdot]\right)\right\|_{T V}
$$

Based on the definitions of distances, mixing time that captures how fast a Markov chain or a hidden Markov chain converges to its stationary state distribution, is defined by

$$
\mathrm{t}(\epsilon) \triangleq \inf \{t \geq 0 \mid \text { for all } \tau>t, \mathrm{~d}(\tau) \leq \epsilon\}
$$

The mixing time of a hidden Markov chain is defined by

$$
\mathrm{t}_{\mathrm{HM}}(\epsilon) \triangleq \inf \left\{t \geq 0 \mid \text { for all } \tau>t, \mathrm{~d}_{\mathrm{HM}}(\tau) \leq \epsilon\right\}
$$

It is straight-forward to see that

$$
\mathrm{t}_{\mathrm{HM}}(\epsilon) \leq \mathrm{t}(\epsilon)
$$

This provides an immediate way to characterize the mixing time of a hidden Markov chain by that of a Markov chain. However, We remark that the mixing time of a hidden Markov chain can be significantly different from that of the underlying Markov chain [6]. Thus it is necessary to directly characterize the mixing time of a hidden Markov chains to obtain accurate characterization.

After introducing mixing time in a general setting, we take $\Omega=\mathcal{S}$ be the set of all feasible states of the CSMA network, $\left\{X_{t}\right\}$ be the Markov chain CSMA-MF and $\tilde{g}=g$ be the link activeness map in this study.

\section{Temporal Starvation}

A detrimental transient phenomenon is temporal starvation, where links can starve for prolonged periods indefinitely often. To characterize temporal starvation, we define some notations.

For any given $b \in \mathcal{B}$, we define

$$
P_{x_{0}}\left(X_{t} \in g^{-1}[b]\right) \triangleq \sum_{x \in \mathcal{S}: g[x]=b} \mathbb{P}\left\{X_{t}=x \mid X_{0}=x_{0}\right\}
$$

Define the temporal throughput of link $v$ at time $t$, starting at initial state $x_{0} \in \mathcal{S}$, as

$$
\mathrm{A}_{x_{0}}^{t}(v) \triangleq \sum_{b \in \mathcal{B}: b(v) \neq 0} P_{x_{0}}\left(X_{t} \in g^{-1}[b]\right)
$$

The stationary throughput of $v$ is given by

$$
\mathrm{T}(v) \triangleq \sum_{b \in \mathcal{B}: b(v) \neq 0} \pi\left(g^{-1}[b]\right)
$$

Note that as the hidden Markov chain converges to its stationary state distribution, the instantaneous throughput $\mathrm{A}_{x_{0}}^{t}(v)$ converges to the stationary throughput $\mathrm{T}(v)$.

We now define a measure of temporal starvation at $v$ within interval $t$ starting with initial state $x_{0}$ by the maximum temporal throughput loss, defined by

$$
\ell(t) \triangleq \max _{v \in V, x_{0} \in \mathcal{S}} \frac{1}{t}\left[\int_{0}^{t}\left(\mathrm{~T}(v)-\mathrm{A}_{x_{0}}^{t^{\prime}}(v)\right) d t^{\prime}\right]^{+},
$$

where $[x]^{+}=\max (x, 0) \cdot \ell(t)$ is a natural measure, which counts the loss from the cumulative throughput a link will receive when starting at a particular state within a certain interval, as compared to the stationary throughput.

Lemma 1. [6]If $t \geq n \cdot \mathrm{t}_{\mathrm{HM}}\left(\frac{\epsilon}{4}\right)$ and $n$ is sufficiently large,

$$
\ell(t) \leq \epsilon
$$

Hence, if we can characterize $\mathrm{t}_{\mathrm{HM}}(\epsilon)$, we can provide bounds on temporal starvation of CSMA-MF. In simulation studies, we usually observe that the regions of fast mixing time imply that of low temporal starvation (e.g., as corroborated in Sec. I-A)

\section{E. Main Problem}

After relating temporal starvation to mixing time (as a tool to understand the simulation results in Sec. I-A), we seek to characterize fast mixing time for the throughput process of CSMA-MF in the rest of the paper.

In the literature of mixing time [7], fast mixing usually refers to mixing time being polynomial in terms of $n$, and oftenly, it is $O\left(n^{r}(\ln n-\ln \epsilon)\right)$. Thus, our goal is to characterize the regions of tuple $(q, \lambda)$ for fast mixing time. Specifically, we use the superscript ${ }^{\text {csma }}$ to denote the mixing time for CSMAMF. For example, let the mixing time for the throughput process of CSMA-MF be $\mathrm{t}_{\mathrm{HM}}^{\mathrm{csma}}(\epsilon)$. We aim at finding the sufficient conditions that

$$
\mathrm{t}_{\mathrm{HM}}^{\mathrm{csma}}(\epsilon) \leq \frac{k_{1}}{\mu_{\mathrm{tx}}+q \cdot \mu_{\mathrm{cd}}} n^{r}(\ln n-\ln \epsilon)
$$

where $k_{1}$ and $r$ are some non-negative constants.

\section{F. Approaches and Results}

The sufficient conditions for fast mixing of CSMA-MF is summarized in Table I. One can see from Table I region 2 to 4 that fast mixing is guaranteed ${ }^{4}$ for $q>2 \Delta, \lambda>1$.

\footnotetext{
${ }^{4}$ The upper bound conditions for $\lambda$ and $q$ in Table I can be relaxed to any other polynomials in $n$ to yield other polynomial upper bounds on mixing time
} 


\begin{tabular}{|c|c|c|c|c|c|}
\hline Region & Conditions on $q$ & Conditions on $\lambda$ & Types of graphs & Bounds on CSMA-MF mixing time & Section \\
\hline 1 & $n>q>\Delta$ & $\lambda<\frac{1}{2 \Delta}$ & Arbitrary & $\mathrm{t}_{\mathrm{HM}}^{\mathrm{csma}}(\epsilon) \leq \frac{4 c}{\left(\mu_{\mathrm{tx}}+q \mu_{\mathrm{cd}}\right)} n(\ln n-\ln \epsilon)$ & Sec. IV-A \\
\hline 2 & $q>\Delta^{2} n \log n$ & $\lambda>1$ & Arbitrary with large $n$ & $\mathrm{t}_{\mathrm{HM}}^{\mathrm{csma}}(\epsilon) \leq \frac{3 c}{\left(\mu_{\mathrm{tx}}+q \mu_{\mathrm{cd}}\right)}\left(\ln n-\ln \frac{\epsilon}{2}\right)$ & Sec. IV-A \\
\hline 3 & $n>q>2 \Delta$ & $\lambda>\frac{4 n^{3} q}{(q-\Delta)(q-2 \Delta) \epsilon}$ & Arbitrary with large $n$ & $\mathrm{t}_{\mathrm{HM}}^{\mathrm{csma}}(\epsilon) \leq \frac{6 c}{\left(\mu_{\mathrm{tx}}+q \mu_{\mathrm{cd}}\right)} n\left(\ln n-\ln \frac{\epsilon}{2}\right)$ & Sec. IV-A \\
\hline 4 & $n \geq q \geq 2 \Delta$ & $\ln n>\lambda>1$ & Arbitrary & $\mathrm{t}_{\mathrm{HM}}^{\mathrm{csma}}(\epsilon) \leq \frac{10 c}{\mu_{\mathrm{tx}}+q \mu_{\mathrm{cd}}} n^{3}(\ln n)^{3}(\ln n-\ln \epsilon)$ & Sec. IV-B \\
\hline \multirow[t]{2}{*}{5} & $n \geq q \geq \Delta+\Delta^{1-\delta}$ & $\ln n>\lambda>1$ & Planar with large $n$ & $\mathrm{t}_{\mathrm{HM}}^{\mathrm{csma}}(\epsilon) \leq \frac{10 c}{\mu_{\mathrm{tx}}+q \mu_{c \mathrm{c}}} n^{3}(\ln n)^{3}(\ln n-\ln \epsilon)$ & Sec. IV-B \\
\hline & $\begin{array}{c}n>q>\left(1+\epsilon_{1}\right) \Delta \\
\quad \text { and } \Delta>(\ln n)^{2}\end{array}$ & $\ln n>\lambda>1$ & $\begin{array}{l}\text { Planar, triangle-free } \\
\text { with large } n\end{array}$ & 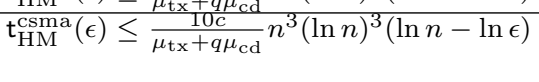 & $\overline{\text { Sec. IV-B }}$ \\
\hline 6 & $\begin{array}{l}\quad n \geq q \geq k_{2} \frac{\Delta}{\ln \Delta} \\
\text { and } \Delta \geq k_{1}(\ln n)^{b_{1}}\end{array}$ & $\ln n>\lambda>1$ & Trees with large $n$ & $\mathrm{t}_{\mathrm{HM}}^{\mathrm{csma}}(\epsilon) \leq \frac{10 c}{\mu_{\mathrm{tx}}+q \mu_{\mathrm{cd}}} n^{3}(\ln n)^{3}(\ln n-\ln \epsilon)$ & Sec. IV-B \\
\hline
\end{tabular}

SUFFICENT CONDITIONS FOR FAST MIXING TIME FOR THE THROUGHPUT PROCESS OF CSMA-MF.

We establish these sufficient conditions by first uniformizing the CSMA-MF (Section III) and then analyzing the discrete time CSMA-MF through a modified approach for path coupling(Section IV-A) and reduction to another Markov chain(Section IV-B). Finally, we end with an example of slow mixing when the number of channels is not sufficient(Section V).

\section{UNIFORMIZATION OF CSMA-MF}

We uniformize CSMA-MF to discrete-time CSMA-MF by taking $\nu=n\left(q \mu_{\mathrm{cd}}+\mu_{\mathrm{tx}}\right)$ as the uniformization constant. Equivalently, discrete-time CSMA-MF $\left\{X_{k}\right\}$ is a Glauber dynamics, whose state transitions from $X_{k}$ to $X_{k+1}$ proceed as follows. Note that $A\left(X_{k}, v\right)$ is the set of available channels at node $v$.

\section{- Discrete-time CSMA-MF:}

1) We first uniformly randomly select a link $v \in V$

2) The new state $X_{k+1}$ agrees everywhere with $X_{k}$ except possibly at $v \cdot X_{k+1}(v)=\tilde{c}$ is updated with probability $p_{\tilde{c}}$ as follows.

a) If $X_{k}(v)=0$, we set

$$
p_{\tilde{c}}= \begin{cases}\frac{\lambda}{(1+q \lambda)} & \text { if } \tilde{c} \in A\left(X_{k}, v\right) \\ \frac{1+\lambda\left(q-\left|A\left(X_{k}, v\right)\right|\right)}{1+\lambda q} & \text { if } \tilde{c}=0 \\ 0 & \text { otherwise }\end{cases}
$$

b) If $X_{k}(v) \neq 0$, we set

$$
p_{\tilde{c}}= \begin{cases}\frac{1}{1+\lambda q} & \text { if } \tilde{c}=0 \\ \frac{\lambda q}{1+\lambda q} & \text { if } \tilde{c}=X_{k}(v) \\ 0 & \text { otherwise }\end{cases}
$$

With notation of having $(\bar{\cdot})$ as the discrete-time quantity (e.g. $\bar{t}_{\mathrm{HM}}$ ), we can relate continuous-time mixing time and discrete-time mixing time as follows.

Lemma 2. [6] If $\bar{t}_{\mathrm{HM}}\left(\frac{\epsilon}{2}\right) \leq k$, then $\mathrm{t}_{\mathrm{HM}}(\epsilon) \leq \frac{k c}{\nu}$, where $c$ is a constant satisfying $\frac{c}{e^{c-1}} \leq \frac{\epsilon}{2}$ and $c \geq 1$.

\section{SUFFICIENT CONDITIONS FOR FAST MIXING}

\section{A. Modified Approach for Path Coupling}

Path coupling is a useful technique to upper bound mixing time of Markov chains and thus to obtain sufficient conditions for fast mixing. However, we study the hidden Markov chain instead of the Markov chain. This requires a different approach of path coupling. Hence, we develop a modified approach of path coupling in order to obtain bounds for the mixing time of the throughput process of CSMA-MF.

1) Coupling and its challenges: Given any two random variables $X$ and $Y$, we are free to construct a joint random variable $Z=(X, Y)$ as long as the marginal probability distribution function in each coordinate in $Z$ is equivalent to the original random variable $X$ or $Y$. Such a construction is called a coupling, which provides a useful way to bound the total variation distance of two random variables.

One can consider one-step maximal coupling (a standard technique in path coupling to bound mixing time in random coloring literature[2]) for discrete-time CSMA-MF to bound $\bar{t}^{\text {csma }}(\epsilon)$. In particular, by applying such coupling, when $\lambda<$ $\frac{1}{\Delta}$, we can show fast mixing for CSMA-MF in region 1 of Table I.

Nonetheless, when $\lambda>\frac{1}{\Delta}$, one cannot obtain similar results [6] using the same method. Therefore, we develop a modified approach for path coupling to work on hidden Markov chain directly, and obtain fast mixing time for the throughput process of CSMA-MF when $\lambda>\frac{1}{\Delta}$.

2) Modified Approach for Path Coupling: First, we define the path metric $(\rho)$ and a new modified path metric $(\tilde{\rho})$ in terms of link activeness. For $x, y \in \mathcal{S}$,

$$
\begin{gathered}
\rho(x, y) \triangleq \sum_{v \in V} \mathbf{1}_{x(v) \neq y(v)} \\
\tilde{\rho}(x, y) \triangleq \sum_{v \in V} \mathbf{1}_{g[x(v)] \neq g[y(v)]}
\end{gathered}
$$

We present a new approach for path coupling to obtain the following results. Our approach utilizes the neighboring configuration of links to construct a coupling between any two initial states.

Theorem 1. [6] With $q>2 \Delta$, suppose that there are two Markov chains $\left\{X_{k}\right\}$ and $\left\{Y_{k}\right\}$ on $\mathcal{S}$ with the same transition probability, but with possibly different initial states distributions $X_{0}, Y_{0}$. If $\exists \alpha>0, \delta \geq 0$ such that the following two conditions are satisfied:

1) There exists a coupling $\left(X_{1}, Y_{1}\right)$ subject to

$$
\mathbb{E}\left[\tilde{\rho}\left(X_{1}, Y_{1}\right) \mid X_{0}, Y_{0}\right] \leq(1-\alpha) \tilde{\rho}\left(X_{0}, Y_{0}\right)
$$


for any pair of $X_{0}, Y_{0}$ differing at only one link $v \in V$ (i.e., $X_{0}(v) \neq Y_{0}(v)$ ) and either $X_{0}(v)=0$ or $Y_{0}(v)=$ 0.

2) There exists a coupling $\left(X_{1}, Y_{1}\right)$ subject to

$$
\mathbb{E}\left[\tilde{\rho}\left(X_{1}, Y_{1}\right) \mid X_{0}, Y_{0}\right] \leq \delta \cdot \rho\left(X_{0}, Y_{0}\right)
$$

for any pair of $X_{0}, Y_{0}$ differing at only one link $v \in V$ (i.e. $X_{0}(v) \neq Y_{0}(v)$ ) and both $X_{0}(v) \neq 0$ and $Y_{0}(v) \neq$ 0.

Then, for all $\left(X_{k}, Y_{k}\right) \in \mathcal{S}^{2}$, there exists a coupling $\left(X_{k+1}, Y_{k+1}\right)$ such that

$$
\begin{aligned}
\mathbb{E}\left[\tilde{\rho}\left(X_{k+1}, Y_{k+1}\right) \mid X_{k}, Y_{k}\right] \leq & (1-\alpha) \tilde{\rho}\left(X_{k}, Y_{k}\right) \\
& +\delta(\Delta+1) \rho\left(X_{k}, Y_{k}\right)
\end{aligned}
$$

Theorem 1 is specifically devised for hidden Markov chains. Furthermore, with an extra condition, we can derive an upper bound for the mixing time of hidden Markov chain $\left\{g\left[X_{k}\right]\right\}$ as follows.

Corollary 1. [6] Given a fixed $\epsilon>0$, if the two conditions of Theorem 1 are satisfied and $\frac{\delta(\Delta+1) n}{\alpha} \leq \frac{\epsilon}{2}$, then

$$
\bar{t}_{\mathrm{HM}}(\epsilon) \leq\left\lceil\frac{\ln n-\ln \frac{\epsilon}{2}}{\alpha}\right\rceil
$$

Using Corollary 1 , we can establish sufficient conditions for fast mixing of the throughput process of CSMA-MF, in the regions of large $q$ and $\lambda$ (i.e. region 2 and 3 in Table I).

\section{B. Reduction to multi-color Glauber dynamics}

We note that directly applying coupling or modified approach of coupling on CSMA-MF cannot offer much insights for region 4 to 6 in Table I. Therefore, we use another approach. We relate the mixing time of CSMA-MF to that of the specific type of multi-color Glauber dynamics, denoted by $\bar{t}^{\mathrm{mcgd}}(\epsilon)$ and show fast mixing of $\bar{t}^{\mathrm{mcgd}}(\epsilon)$ in region 4 to 6.

We define a specific type of multi-color Glauber dynamics related to CSMA networks, whose state transitions from $X_{k}$ to $X_{k+1}$ proceed as follows.

\section{- Multi-color Glauber Dynamics:}

1) We first uniformly randomly select a link $v \in V$.

2) The new state $X_{k+1}$ agrees everywhere with $X_{k}$ except possibly at $v \cdot X_{k+1}(v)=\tilde{c}$ is updated with probability $p_{\tilde{c}}$ as follows.

$$
p_{\tilde{c}}= \begin{cases}\frac{\lambda}{1+\left(\left|A\left(X_{k}, v\right)\right|\right) \lambda}, & \text { if } \tilde{c} \in A\left(X_{k}, v\right) \\ \frac{1}{1+\left(\left|A\left(X_{k}, v\right)\right|\right) \lambda}, & \text { if } \tilde{c}=0 \\ 0 & \text { otherwise }\end{cases}
$$

By suitably defining a canonical path between these two Markov chains, one can show,

Theorem 2. [6] On arbitrary conflict graph, if $q>\Delta$ and $\lambda>1$, we have

$$
\bar{t}^{\text {csma }}(\epsilon) \leq \bar{t}^{\mathrm{mcgd}}(\epsilon) \frac{2 \lambda^{2} q(1+\lambda q)}{1+(q-\Delta) \lambda} \frac{\ln \left(\frac{1}{\epsilon}\right)+n \ln (1+q \lambda)}{\ln \frac{1}{2 \epsilon}}
$$

Therefore, whenever multi-color Glauber dynamics has fast mixing time, CSMA-MF also has fast mixing time. In [6], we characterize the sufficient conditions for fast mixing time for multi-color Glauber dynamics by upper bounding $\bar{t}^{\mathrm{mcgd}}(\epsilon)$.

\section{Necessary CONDitions for Fast Mixing}

To complete the story on characterizing the region for fast mixing time of $\bar{t}_{\mathrm{HM}}^{\mathrm{csma}}(\epsilon)$, we also investigate the lower bound of mixing time to obtain necessary conditions for fast mixing of hidden Markov chains. We note that this study is nontrivial because we cannot use the mixing time of a Markov chain to obtain a lower bound for that of a hidden Markov chain.Therefore, we develop new tools, for examples, by generalizing Cheeger's constant to establish lower bounds. Given $H \subset g(\mathcal{S})$, with $Q(\tilde{A}, \tilde{B}) \triangleq \sum_{x \in \tilde{A}} \sum_{y \in \tilde{B}} \pi(x) P(x, y)$, we define the modified Cheeger's constant as

$$
\Phi_{g^{-1}}^{*}(\epsilon)=\min _{H \subset \mathcal{S} \mid \pi\left(g^{-1}(H)\right) \leq 1-2 \epsilon} \frac{Q\left(g^{-1}(H), g^{-1}\left(H^{c}\right)\right)}{\pi\left(g^{-1}(H)\right)}
$$

We claim that the mixing time for the throughput process is bounded below by,

Theorem 3. [6] $\quad \bar{t}_{\mathrm{HM}}(\epsilon) \geq \frac{\epsilon}{\Phi_{g^{-1}}^{*}(\epsilon)}$

Applying this, one can show that for a complete bipartite conflict graph when $\lambda>1$ and $q<<\Delta$, $\bar{t}_{\mathrm{HM}}^{\mathrm{csma}}\left(\frac{1}{8}\right)$ is exponential in $n$. Hence, this corroborates with our simulation results that temporal starvation can be severe if the number of frequency channels is insufficient.

\section{CONCLUSION}

We study the effect of number of frequency channels on temporal starvation in general CSMA networks. We investigate this via mixing time analysis of a hidden Markov chain. This paper presents sufficient and necessary conditions for fast mixing of the corresponding hidden Markov chain, which allows us to understand the simulation observations.

\section{REFERENCES}

[1] M. Durvy, O. Dousse, and P. Thiran. On the fairness of large CSMA networks. In IEEE J. Selected Areas in Communications, volume 27, pages 1093-1104, Sep. 2009.

[2] A. Frieze and E. Vigoda. A survey on the use of markov chains to randomly sample colorings. In Combinatorics, Complexity and Chance, A tribute to Dominic Welsh, pages 53-71. Oxford University Press, 2007.

[3] L. Jiang, M. Leconte, J. Ni, R. Srikant, and J. Walrand. Fast mixing of parallel glauber dynamics and low-delay CSMA scheduling. In Proc. IEEE INFOCOM Mini-Conference, 2011.

[4] L. B. Jiang and J. Walrand. A distributed CSMA algorithm for throughput and utility maximization in wireless networks. IEEE/ACM Trans. Networking, 18(3):960-97, Jun. 2010.

[5] C. H. Kai and S. C. Liew. Temporal starvation in CSMA wireless networks. In Proc. IEEE ICC, 2011.

[6] K.-K. Lam, C.-K. Chau, M. Chen, and S.-C. Liew. On the mixing time and temporal starvation of CSMA networks with multiple frequency agility. Technical report, 2011. Available at http://staff.ie.cuhk.edu.hk/ mhchen/ papers/csmamixing.pdf.

[7] D. A. Levin, Y. Peres, and E. L. Wilmer. Markov chains and Mixing Times. AMS, 2009.

[8] S. C. Liew, J. Zhang, C.-K. Chau, and M. Chen. Analysis of frequencyagile CSMA networks. Technical report, 2010. Available at Arxiv: http: //arxiv.org/1007.5255. 